\title{
Strain effects on phonon transport in antimonene from a first-principles study
}

\author{
Ai-Xia Zhang, Jiang-Tao Liu and San-Dong Guo \\ School of Physics, China University of Mining and Technology, Xuzhou 221116, Jiangsu, China
}

\begin{abstract}
Strain engineering is a very effective method to continuously tune the electronic, topological, optical and thermoelectric properties of materials. In this work, strain-dependent phonon transport of recently-fabricated antimonene (Sb monolayer) under biaxial strain is investigated from a combination of first-principles calculations and the linearized phonon Boltzmann equation within the single-mode relaxation time approximation (RTA). It is found that the ZA dispersion of antimonene with strain less than $-1 \%$ gives imaginary frequencies, which suggests that compressive strain can induce structural instability. Experimentally, it is possible to enhance structural stability by tensile strain. Calculated results show that lattice thermal conductivity increases with strain changing from $-1 \%$ to $6 \%$, and lattice thermal conductivity at $6 \%$ strain is 5.6 times larger than that at $-1 \%$ strain at room temperature. It is interesting that lattice thermal conductivity is in inverse proportion to buckling parameter $h$ in considered strain range. Such a strain dependence of lattice thermal conductivity is attributed to enhanced phonon lifetimes caused by increased strain, while group velocities have a decreased effect on lattice thermal conductivity with increasing strain. It is found that acoustic branches dominate the lattice thermal conductivity over the full strain range. The cumulative room-temperature lattice thermal conductivity at $-1 \%$ strain converges to maximum with phonon mean free path (MFP) at $50 \mathrm{~nm}$, while one at $6 \%$ strain becomes as large as $44 \mu \mathrm{m}$, which suggests that strain can give rise to very strong size effects on lattice thermal conductivity in antimonene. These results may provide guidance on fabrication techniques of antimonene, and offer perspectives on tuning lattice thermal conductivity by size and strain for applications of thermal management and thermoelectricity.
\end{abstract}

PACS numbers: 72.15.Jf, 71.20.-b, 71.70.Ej, 79.10.-n Email:guosd@cumt.edu.cn Keywords: Strain; Lattice thermal conductivity; Group velocities; Phonon lifetimes

\section{INTRODUCTION}

Due to their novel physical and chemical properties, two-dimensional (2D) materials have been widely investigated both theoretically and experimentally, including semiconducting transition-metal dichalcogenide ${ }^{1}$, group IV-VI ${ }^{2}$, group-VA ${ }^{3,4}$ and group-IV ${ }^{5}$ monolayers. Graphene, as representative of these monolayers, has a unique massless Dirac-like electronic excitation ${ }^{6}$, and has extremely high lattice thermal conductivity ${ }^{7}$. In comparison with the gapless graphene, $\mathrm{MoS}_{2}$ is another classic 2D monolayer with intrinsic direct band gap of $1.9 \mathrm{eV}$, which has been applied in field effect transistors, photovoltaics and photocatalysis ${ }^{8-11}$. Recently, semiconducting group-VA elements (As, Sb, Bi) monolayers with the graphene-like buckled structure are predicted to be of good stability by the first-principle calculations ${ }^{3}$. Subsequently, Sb monolayer (antimonene) of them is successfully synthesized on various substrates via van der Waals epitaxy growth ${ }^{12,13}$. Experimentally, graphene-like buckled structure is confirmed for antimonene by Raman spectroscopy and transmission electron microscopy ${ }^{12,13}$.

In theory, thermal transport of antimonene has been widely investigated, including electron and phonon parts $^{14-17}$. It has been predicted that chemical functionalization can effectively reduce lattice thermal conductivity of antimonene ${ }^{16}$. The lattice thermal conductivity of antimonene is predicted to be $15.1 \mathrm{Wm}^{-1} \mathrm{~K}^{-114}$, $13.8 \mathrm{Wm}^{-1} \mathrm{~K}^{-115}$ and $2.3 \mathrm{Wm}^{-1} \mathrm{~K}^{-116}$ based on firstprinciples calculations and the phonon Boltzmann equation. A remarkable discrepancy may be because the dif-
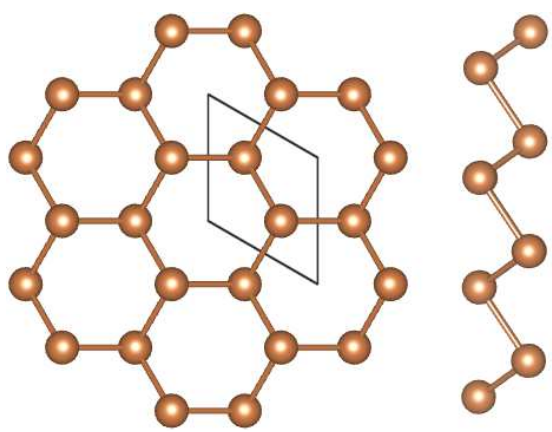

FIG. 1. (Color online) Top and side view of the crystal structure of antimonene, and the frame surrounded by a black box is unit cell.

ferent thickness of antimonene is adopted to calculate lattice thermal conductivity. Recently, we have systematically investigated the thermoelectric properties of groupVA elements (As, Sb, Bi) monolayers ${ }^{17}$, and the lattice thermal conductivity from As to Bi monolayer decreases by using sheet thermal conductance for a fair comparison. Strain can effectively tune the intrinsic physical properties of 2D materials, such as electronic and thermoelectric properties. Semiconductor-metal phase transition can be induced in $\mathrm{MoS}_{2}$ monolayer by applied strain, and strain can also lead to significantly enhanced power factor ${ }^{18,19}$. For $\mathrm{PtSe}_{2}$ and $\mathrm{ZrS}_{2}$, tensile strain can induce reduced lattice thermal conductivity ${ }^{20,21}$. Tensile strains can induce strong size effects for lattice thermal conductivities of silicene, germanene and stanene, and their lattice thermal 

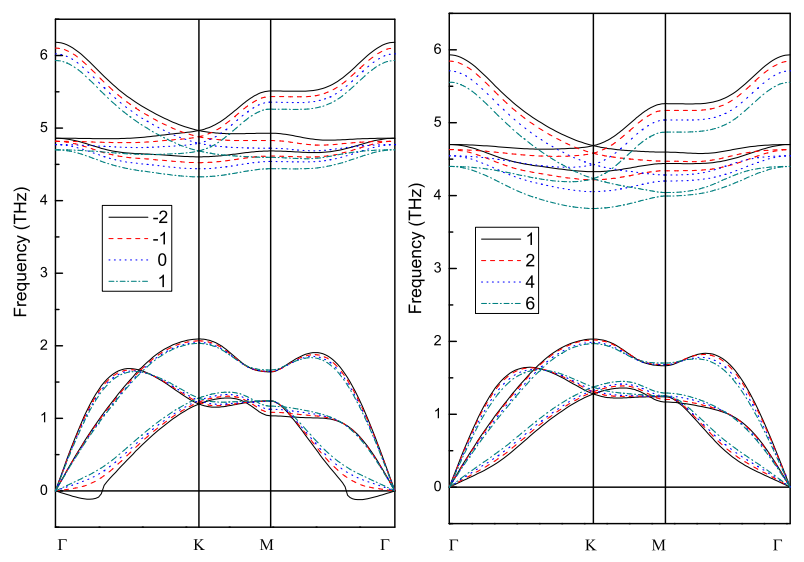

FIG. 2. (Color online) Phonon band structures of antimonene with strain from $-2 \%$ to $6 \%$.

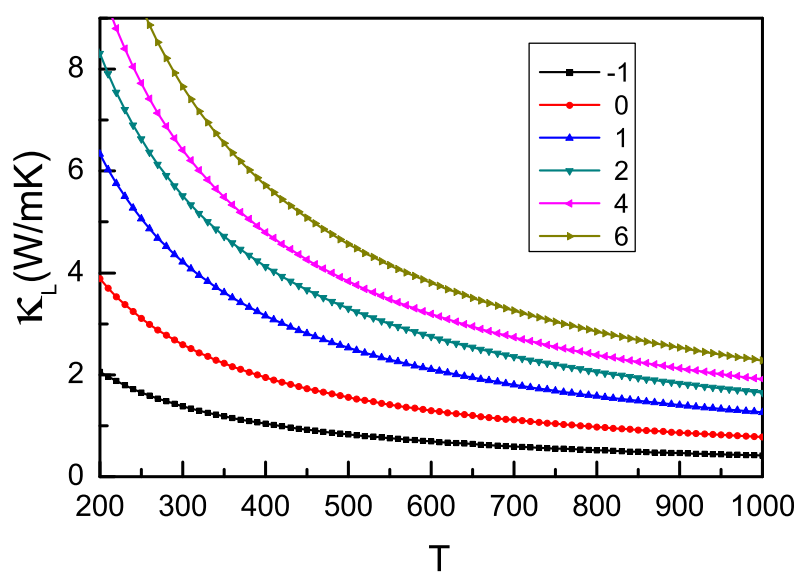

FIG. 3. (Color online) The lattice thermal conductivities of antimonene with strain from $-1 \%$ to $6 \%$ as a function of temperature.

conductivities firstly increase with increasing strain, and then decrease with further strain ${ }^{22,23}$. Phonon transport in both strained and unstrained graphene has been investigated by a rigorous first principles Boltzmann-Peierls equation, and the out-of-plane acoustic branch provides the dominant contribution to lattice thermal conductivity of graphene ${ }^{24}$. In real applications, the residual strain usually exists in nanoscale devices ${ }^{25}$, and the conduction of heat plays a important role in real-world devices. Therefore, it is interesting and necessary to investigate strain effects on phonon transport in recently-fabricated antimonene.

In this work, the strain-dependent phonon transport in antimonene is investigated based on the single-mode RTA. It is found that compressive strain can produce structural instability in antimonene, while tensile strain can enhance structural stability. The lattice thermal conductivity increases with increasing strain, which is in inverse proportion to buckling parameter $h$. The tensilestrain enhanced lattice thermal conductivity is due to improved phonon lifetimes caused by increased strain.

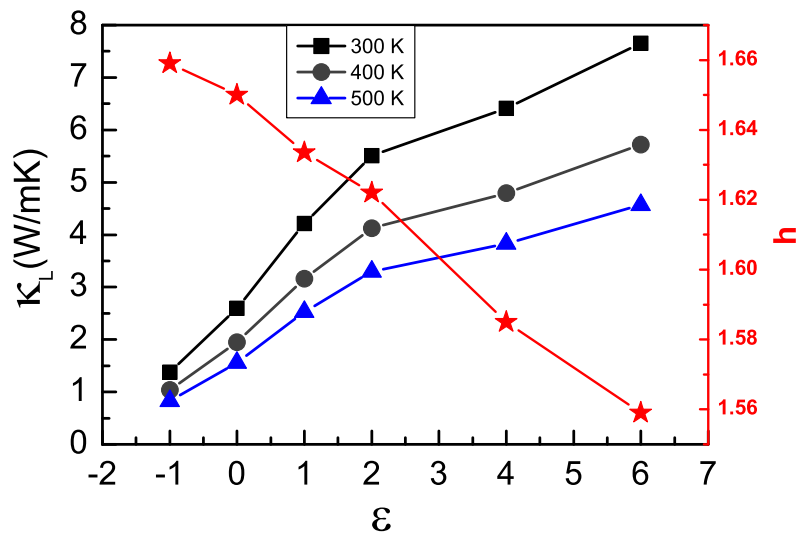

FIG. 4. (Color online) The lattice thermal conductivities (300, 400 and $500 \mathrm{~K})$ and buckling parameter $h(\AA)$ of antimonene versus strain $\varepsilon$.

The strain can induce very strong size effects on lattice thermal conductivity in antimonene, indicating the possibility to manipulate thermal management by strain and size.

The rest of the paper is organized as follows. In the next section, we shall give our computational details about the first-principle and phonon transport calculations. In the third section, we shall present strain effect on phonon transport of antimonene. Finally, we shall give our conclusions in the fourth section.

\section{COMPUTATIONAL DETAIL}

First-principles calculations are performed within projector augmented-wave method and the generalized gradient approximation (GGA) exchange-correlation functional of Perdew-Burke-Ernzerhof (PBE), as implemented in the VASP code ${ }^{26-29}$. The unit cell of antimonene is built with the vacuum region of larger than 16 $\AA$ to avoid spurious interaction. The electronic stopping criterion is $10^{-8} \mathrm{eV}$. The lattice thermal conductivity of antimonene is carried out with the single mode RTA and linearized phonon Boltzmann equation using Phono3py $\operatorname{code}^{30}$. The second order harmonic and third order anharmonic interatomic force constants (IFC) are calculated by using a $5 \times 5 \times 1$ supercell and a $4 \times 4 \times 1$ supercell, respectively. Using the harmonic IFCs, phonon dispersion can be attained by Phonopy package ${ }^{31}$, which determines the allowed three-phonon scattering processes, and further the group velocity and specific heat can be calculated. Based on third-order anharmonic IFCs, the three-phonon scattering rate can be calculated, which determines the phonon lifetimes. To compute lattice thermal conductivities, the reciprocal spaces of the primitive cells are sampled using the $50 \times 50 \times 2$ meshes. For $2 \mathrm{D}$ material, the calculated lattice thermal conductivity depends on the length of unit cell used in the calculations along $\mathrm{z}$ direction ${ }^{32}$. The lattice thermal conductiv- 

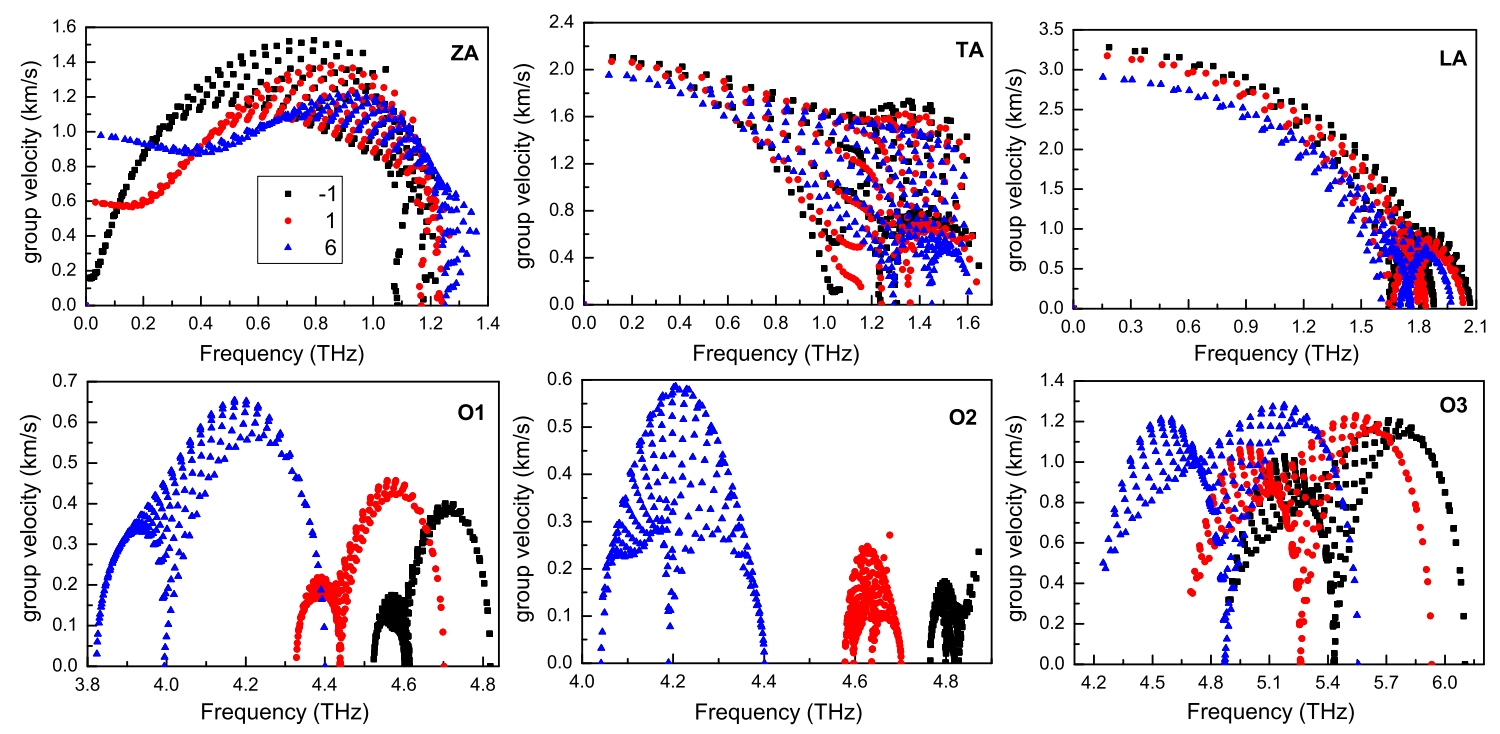

FIG. 5. (Color online) The phonon mode group velocities of antimonene with strain being $-1 \%, 1 \%$ and $6 \%$ in the first Brillouin zone.

ity should be normalized by multiplying $\mathrm{Lz} / \mathrm{d}$, in which $\mathrm{Lz}$ is the length of unit cell along $\mathrm{z}$ direction and $\mathrm{d}$ is the thickness of $2 \mathrm{D}$ material, but the d is not well defined. In this work, the length of unit cell (18 A) along z direction is used as the thickness of antimonene. To make a fair comparison between various 2D monolayers, the thermal sheet conductance can be used, defined as $\kappa \times \mathrm{d}$.

\section{MAIN CALCULATED RESULTS AND ANALYSIS}

The antimonene ( $\beta$-phase) possesses a graphenelike buckled honeycomb structure ${ }^{3,12,13}$, which is shown in Figure 1. Similar structure configuration can be found in silicene, germanene, and stanene with a small buckling ${ }^{22,23}$. The $\varepsilon=\left(a-a_{0}\right) / a_{0}$ is defined to describe biaxial strain, in which $a_{0}$ is the unstrain lattice constant. The calculations are performed on nine values of $\varepsilon$ ranging from $-6 \%$ to $6 \%$, and the $\varepsilon<0(>0)$ means compressive (tensile) strain. Firstly, we calculate the phonon dispersion in high symmetry directions with strain from $-6 \%$ to $6 \%$. Calculated results show that the phonon dispersions give imaginary frequencies with strain less than $-1 \%$, which indicates that these freestanding antimonene are not stable. These results may suggest that tensile strain can strengthen the stability of antimonene in experiment. Similar results also can be found in germanene, and stanene, and tensile strain can harden soft modes ${ }^{23}$. Here, only phonon dispersion curves with strain from $2 \%$ to $6 \%$ are plotted in Figure 2. Although out-of-plane acoustic modes of antimonene have coupling with the inplane longitudinal acoustic (LA) and transversal acoustic (TA) modes due to buckling, they are still marked with ZA modes. It is clearly seen that the ZA disper- sion at $-2 \%$ strain shows imaginary frequencies near the $\Gamma$ point, and the imaginary frequencies become large with increasing compressive strain. The optical branches overall move downward with increasing strain from $-1 \%$ to $6 \%$, which can be explained by the reduction of the material stiffness. These phonon softening for optical branches are similar to those in flat graphene and buckled silicene, germanene and stanene ${ }^{23}$. The phonon band gap decreases from $2.46 \mathrm{THz}$ to $1.86 \mathrm{THz}$ with strain changing from $-1 \%$ to $6 \%$. It is found that the slope of the ZA branch increases with increasing strain, while the ones of LA and TA branches decrease. Moreover, the slope of ZA branch changes more significantly than ones of LA and TA branches. It is found that dispersion of LA and TA modes of antimonene changes slightly with increasing strain, which is comparable with silicene, germanene, and stanene, but is different from graphene $\mathrm{e}^{22,23}$.

The lattice thermal conductivities of antimonene in the strain range of $-1 \%-6 \%$ are calculated with RTA method, and they as a function of temperature are plotted Figure 3. Calculated results predict that the lattice thermal conductivity increases with strain increasing in considered strain range. To study changing speed of lattice thermal conductivity, the lattice thermal conductivities as a function of strain at the temperature of 300 , 400 and $500 \mathrm{~K}$ are shown in Figure 4. The lattice thermal conductivity first increases rapidly with strain being less than $2 \%$, and then rises more slowly. It is predicted that lattice thermal conductivities of silicene, germanene, and stanene show similar increase with increasing strain within certain strain range ${ }^{22,23}$, and the lattice thermal conductivities decrease, when the strain is greater than critical value. The strain dependence of lattice thermal conductivity of antimonene is different from those of monolayer $\mathrm{PtSe}_{2}$ and $\mathrm{ZrS}_{2}{ }^{20,21}$. From As to Bi mono- 

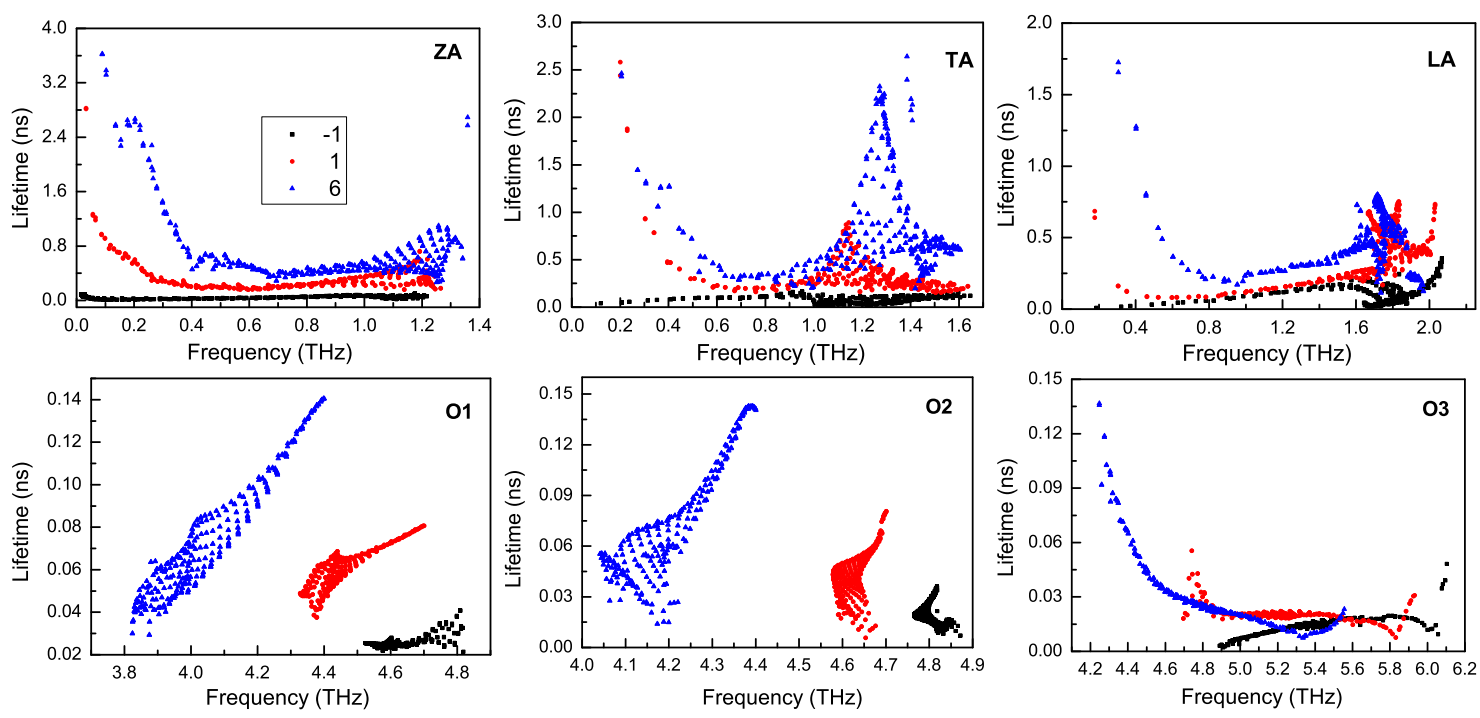

FIG. 6. (Color online) The phonon mode lifetimes of antimonene with strain being $-1 \%, 1 \%$ and $6 \%$ in the first Brillouin zone.

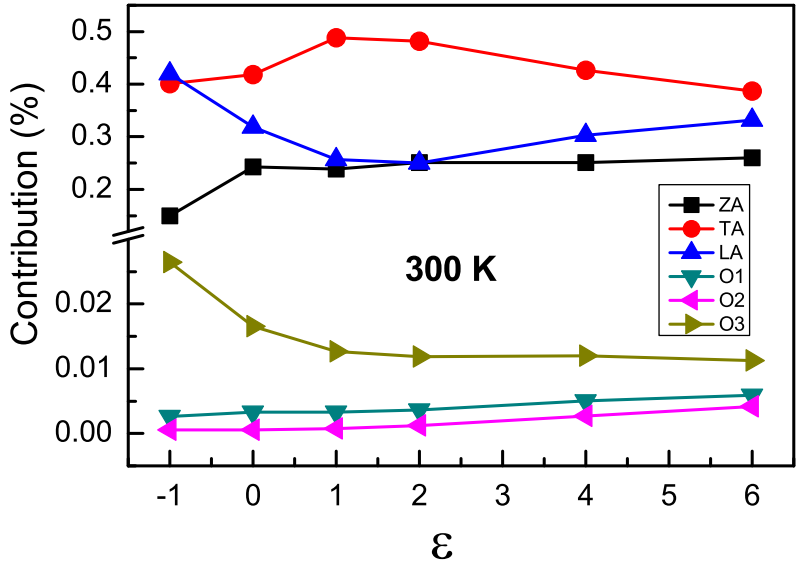

FIG. 7. (Color online) phonon modes contributions to total lattice thermal conductivity as a function of strain $\varepsilon(300 \mathrm{~K})$.

layer, the lattice thermal conductivity decreases, and the corresponding buckling parameter $h$ increases ${ }^{17}$. The lattice thermal conductivity should have a correlation with the buckling parameter, and larger buckling parameter $h$ means lower lattice thermal conductivity. Therefore, the buckling parameter $h$ as a function of strain is also plotted in Figure 4. With increasing strain from -1\% to 6\%, the buckling parameter $h$ decreases, and then incremental lattice thermal conductivity is produced. Similar relation between lattice thermal conductivity and buckling parameter can also be found in silicene ${ }^{22}$. This may apply to other 2D monolayers with intrinsic buckling, such as germanene, stanene, As and Bi monolayers. Moreover, As-As bond length keeps increasing with strain becoming larger. The decreasing buckling parameter $h$ and increasing As-As bond length mean that tensile strain can make the structure of antimonene become more planar.

To identify the underlying mechanism of strain- dependent lattice thermal conductivity, phonon mode group velocities of antimonene are calculated, using the harmonic IFCs, which are plotted in Figure 5, with -1\%, $1 \%$ and $6 \%$ strains. For ZA branch, the largest group velocity near $\Gamma$ point increases from $0.16 \mathrm{kms}^{-1}$ to 0.59 $\mathrm{kms}^{-1}$ to $0.98 \mathrm{kms}^{-1}$ with strain changing from $-1 \%$ to $1 \%$ to $6 \%$. This can be understood by increased stiffness in $\mathrm{Z}$ direction caused by increased strain in $\mathrm{X}$ and $\mathrm{Y}$ directions. The largest group velocity of TA (LA) near $\Gamma$ point decreases from $2.11(3.28) \mathrm{kms}^{-1}$ for $-1 \%$ strain to 2.07 (3.17) $\mathrm{kms}^{-1}$ for $1 \%$ strain to $1.95(2.90) \mathrm{kms}^{-1}$ for $6 \%$ strain, which is due to weakened As-As interatomic interaction in $\mathrm{X}$ and $\mathrm{Y}$ directions caused by strain-increased As-As bond length. These results agree well with change of the slope of ZA, TA and LA branches caused by increased strain. Most of group velocities for ZA branch become small with increasing strain, and group velocities for TA branch have small changes, and group velocities for LA branch change slightly small. These straindependent group velocities should lead to decrescent lattice thermal conductivity with increasing strain. The group velocities of optical branches increase with strain increasing, especially for first two optical branches, which can produce larger lattice thermal conductivity, but the optical branches have little contribution to total lattice thermal conductivity (hereinafter discussed). Therefore, the changes of group velocities caused by increasing strain should make opposite contribution to increased lattice thermal conductivity.

Next, we consider strain-dependent phonon lifetimes, which are merely proportional to lattice thermal conductivity in the single-mode RTA method ${ }^{30}$. The phonon lifetimes can be calculated by three-phonon scattering rate from third-order anharmonic IFCs. The phonon lifetimes with $-1 \%, 1 \%$ and $6 \%$ strains are shown in Figure 6 . For all phonon branches, most of phonon lifetimes 


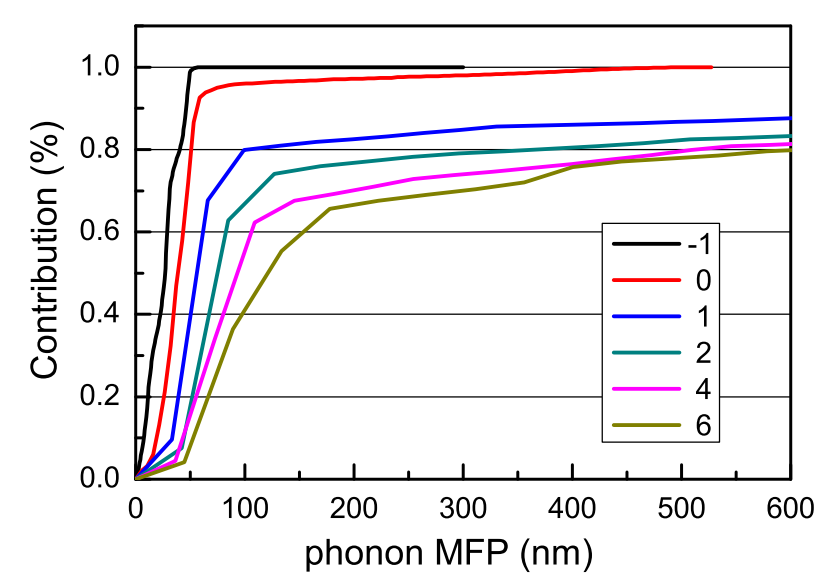

FIG. 8. (Color online) Cumulative lattice thermal conductivity divided by total lattice thermal conductivity with respect to phonon mean free path at room temperature.

become large with increasing strain, which can induce large lattice thermal conductivity. It is clearly seen that acoustic branches have very larger group velocities and phonon lifetimes than optical ones, which means that acoustic branches dominate the lattice thermal conductivity. Therefore, we only consider group velocities and phonon lifetimes of acoustic branches to find out the underlying mechanism of enhanced lattice thermal conductivity caused by increased strain. The strain dependence of group velocities has decreased effect on lattice thermal conductivity, while strain dependence of phonon lifetimes has enhanced influence. So, the improved phonon lifetimes with increasing strain lead to strain-dependent lattice thermal conductivity. Similar mechanism can be found in silicene, germanene, and stanene, and the acoustic phonon lifetimes determine their strain dependence of lattice thermal conductivity ${ }^{22,23}$.

To further understand strain dependence of lattice thermal conductivity, the relative contributions of acoustic and optical branches to the total lattice thermal conductivity as a function of strain are plotted Figure 7. It is clearly seen that the acoustic modes give the dominant contribution, while contribution from optical branches is $1.7 \%-3.0 \%$. In considered strain range, the ZA branch has the smallest contribution among acoustic branches except $2 \%$ strain, and the TA branch has the largest contribution but - $1 \%$ strain. The LA and TA branches contribute more than $71 \%$ of the total thermal conductivity, up to $82 \%$ especially for $-1 \%$ strain. This is the same with silicene $^{22}$, but is quite different from graphene, for which ZA branch has a major contribution ${ }^{24}$. The contribution of ZA mode first increases from $-1 \%$ to $0 \%$ strain, and then remains about the same. The lattice thermal conductivity contributed by the TA mode first increases from $-1 \%$ to $1 \%$ strain, and then decreases. However, the contribution of LA mode with strain up to $2 \%$ first decreases, and then increases. The contribution from the third optical branch first rapidly decreases from $-1 \%$ to $2 \%$ strain, and then slowly decreases from $2 \%$ to $6 \%$ strain. The contributions of first two optical modes keep increasing tendency from $-1 \%$ to $6 \%$ strain.

To study size effect on lattice thermal conductivity, cumulative lattice thermal conductivity divided by total lattice thermal conductivity with respect to MFP, at room temperature, are plotted in Figure 8, with strain from $-1 \%$ to $6 \%$. With MFP increasing, the cumulative lattice thermal conductivity increases, and then approaches maximum. The corresponding MFP of maximum changes from $50 \mathrm{~nm}$ to $43590 \mathrm{~nm}$ with strain from $-1 \%$ to $6 \%$. This means that lattice thermal conductivity is contributed by phonons with longer MFP, as the strain becomes larger. The critical MFP at $6 \%$ strain is 868 times larger than that at $-1 \%$ strain, which demonstrates that strain induces very strong size effects for lattice thermal conductivity of antimonene. The strong size effects on lattice thermal conductivity caused by strain can also be found in in silicene, germanene, and stanene ${ }^{23}$. The scale reduction may be an effective method to reduce lattice thermal conductivity for antimonene with large strain. When the lattice thermal conductivity is reduced to $60 \%$ by nanostructures, the characteristic length changes from $29 \mathrm{~nm}$ to $155 \mathrm{~nm}$ with strain from $-1 \%$ to $6 \%$.

\section{CONCLUSION}

In summary, the lattice thermal conductivity of antimonene under strain is performed by the first-principles calculations and linearized phonon Boltzmann equation within the single-mode RTA. It is found that increasing strain can harden and stabilize long wavelength ZA acoustic branch. This may provide guidance on fabrication of As and Bi monolayers by tensile strain. From $-1 \%$ to $6 \%$ strain, the lattice thermal conductivity of antimonene increases, which is mainly due to the straindependent phonon lifetimes. The phonon lifetimes increase with increasing strain, which leads to enhanced lattice thermal conductivity. Strain can induce a strong size effect on lattice thermal conductivity in antimonene, which means that the lattice thermal conductivity can be effectively tuned by size and strain. This work provides insight into strain dependence of phonon transport in antimonene, and As and Bi monolayers may have similar strain dependence of lattice thermal conductivity.

\section{ACKNOWLEDGMENTS}

This work is supported by the National Natural Science Foundation of China (Grant No. 11404391). We are grateful to the Advanced Analysis and Computation Center of CUMT for the award of CPU hours to accomplish this work. 
1 M. Chhowalla, H. S. Shin, G. Eda, L. J. Li, K. P. Loh and H. Zhang, Nature Chemistry 5, 263 (2013).

2 R. X. Fei, W. B. Li, J. Li and L. Yang, Appl. Phys. Lett. 107, 173104 (2015).

3 S. L. Zhang et al., Angew. Chem. 128, 1698 (2016).

4 J. P. Ji et al., Nat. Commun. 7, 13352 (2016).

5 S. Balendhran, S. Walia, H. Nili, S. Sriram and M.Bhaskaran, small 11, 640 (2015).

6 A. H. CastroNeto, F. Guinea, N. M. R. Peres, K. S. Novoselov, A. K. Geim, Rev. Mod. Phys. 81, 109 (2009).

7 A. A. Balandin, S. Ghosh, W. Bao, I. Calizo, D. Teweldebrhan, F. Miao, and C. N. Lau, Nano Lett. 8, 902 (2008).

8 K. F. Mak, C. Lee, J. Hone, J. Shan, and T. F. Heinz, Phys. Rev. Lett. 105, 136805 (2010).

9 D. J. Late, B. Liu, H. R. Matte, V. P. Dravid, C. N. R. Rao, Acs Nano 6, 5635 (2012).

10 B. Radisavljevic, A. Radenovic, J. Brivio, V. Giacometti and A. Kis, Nature Nanotechnology 6, 147 (2011).

11 X. Zong et al. J. Am. Chem. Soc. 130, 7176 (2008).

12 P. Ares et al., Adv. Mater. 28, 6332 (2016).

13 J. P. Ji et al., Nat. Commun. 7, 13352 (2016).

14 S. D. Wang, W. H. Wang and G. J. Zhao, Phys. Chem. Chem. Phys. 18, 31217 (2016)

15 G. H. Zheng, Y. L. Jia, S. Gao and S. H. Ke, Phys. Rev. B 94, 155448 (2016).

16 T. Zhang, Y. Y. Qi, X. R. Chen and L. C. Cai, Phys. Chem. Chem. Phys. 18, 30061 (2016).
17 D. C. Zhang, A. X. Zhang and S. D. Guo, arXiv:1701.08944 (2017).

18 E. Scalise, M. Houssa, G. Pourtois, V. Afanas'ev and A. Stesmans, Nano Res. 5, 43 (2012).

19 S. D. Guo, Comp. Mater. Sci. 123, 8 (2016).

20 S. D. Guo, J. Mater. Chem. C 4, 9366 (2016).

${ }^{21}$ H. Y. Lv, W. J. Lu, D. F. Shao, H. Y. Lub and Y. P. Sun, J. Mater. Chem. C 4, 4538 (2016).

${ }^{22}$ H. Xie, T. Ouyang, E. Germaneau, G. Z. Qin, M. Hu and H. Bao, Phys. Rev. B 93, 075404 (2016).

${ }^{23}$ Y. D. Kuang, L. Lindsay, S. Q. Shic and G. P. Zheng, Nanoscale, 8, 3760 (2016).

24 L. Lindsay, Wu Li, J. Carrete, N. Mingo, D. A. Broido and T. L. Reinecke, Phys. Rev. B 89, 155426 (2014).

25 S. Bhowmick and V. B. Shenoy, J. Chem. Phys. 125, 164513 (2006).

${ }^{26}$ G. Kresse, J. Non-Cryst. Solids 193, 222 (1995).

27 G. Kresse and J. Furthmüller, Comput. Mater. Sci. 6, 15 (1996).

28 G. Kresse and D. Joubert, Phys. Rev. B 59, 1758 (1999).

29 J. P. Perdew, K. Burke and M. Ernzerhof, Phys. Rev. Lett. 77, 3865 (1996)

30 A. Togo, L. Chaput and I. Tanaka, Phys. Rev. B 91, 094306 (2015).

31 A. Togo, F. Oba, and I. Tanaka, Phys. Rev. B 78, 134106 (2008).

32 X. F. Wu, V. Varshney et al., Chem. Phys. Lett. 669, 233 (2017). 